\title{
Investigations on gradient a.c. conductivity characteristics of bamboo (Dendrocalamus strictus)
}

\author{
NAVIN CHAND*, DEEPAK JAIN and ARCHANA NIGRAWAL \\ Regional Research Laboratory (CSIR), Bhopal 462 026, India
}

MS received 7 May 2005; revised 30 January 2006

\begin{abstract}
Effect of temperature and frequency variation on a.c. conductivity of bamboo was determined by using a 4274 A Multi-Frequencies LCR meter. Electrical measurements were carried out in the temperature range $24-120^{\circ} \mathrm{C}$ and in the frequency range $4-100 \mathrm{kHz}$. It was observed that the a.c. conductivity increased initially and then decreased with increase of temperature and frequencies. The increase of distance from outer surface to the inner surface side increased the a.c. conductivity values and showed the grading in a.c. conductivity behaviour. Two phases of a.c. conductivity behaviour with temperature exist in bamboo. At $10 \mathrm{~mm}$ distance a.c. conductivity suddenly increases which is the critical depth from skin for this bamboo. Increase of temperature, at all the frequencies increases the a.c. conductivity initially and then decreases. Downward peaks in a.c. conductivities are observed at all the frequencies due to the presence of moisture in bamboo, which liberated on heating. Sharp peak is observed in case of sample 4, which is inner most strip. Maximum sharp peak is observed at lowest $4 \mathrm{kHz}$ frequency.
\end{abstract}

Keywords. a.c. conductivity; bamboo; gradient.

\section{Introduction}

Bamboo is a natural lignocellulosic based composite material and is an abundant natural resource in Asia and South America, and has been used traditionally for fabrication of village houses as a structural material. Massive use of bamboo is possible if it can be used as a reinforcing constituent in a composite material (Shin 1989).

Different types of lignocellulosic and fibrous materials such as wood and bamboo are being increasingly used for insulation and lamination applications by using different polymers such as methylmethacrylate (MMA) and butyl methylacrylate (BMA). Electrical properties like resistivity and dielectric constant of wood have been reported with different moisture contents in the past (Khan et al 1991). It has been found that dielectric constant of wood increased significantly with moisture content. Another important observation that the dielectric constants of untreated wood also increased with density (Chia et al 1986) was reported.

Variation of dielectric constant with moisture content is reported in literature and it was found that all untreated woods had higher dielectric constant than their polymer composites (Chia et al 1986). They concluded that the presence of polymers led to decrease in the number of polarizable units to use bamboo for insulating applications.

Among the natural fibres (Kulkarni et al 1983; White and Ansell 1983; Roe and Ansell 1985), which have been

*Author for correspondence (navinchand15@indiainfo.com) used as reinforcement in plastics, bamboo has low density (Jindal 1986) and high mechanical strength (Shah and Lakkad 1981; Jain et al 2005). Specific gravity of bamboo is $\sim 0.66$ as compared to glass, which is $\sim 2.5$. The reported specific tensile strength of bamboo fibres $\left(0.28 \mathrm{GNm}^{-2}\right)$ is less than that of glass fibres, which is $1.3 \mathrm{GNm}^{-2}$.

Over the past decade natural fibres have found use as potential resources for making low cost composite material. Composites as a dielectric are becoming more popular and studies of electrical properties of natural fibre reinforced polymer composites are, therefore, very important. The electrical properties of some natural fibres, such as volume resistivity and dielectric strength have been studied by Kulkarni et al (1981).

Knowledge of a.c. conductivity behaviour of bamboo from outer side of stem wall to centre of the bamboo is indispensible for finding its proper application. In this paper, strips of bamboo have been cut from the centre to the outer periphery side. A.C. conductivity values have been determined at different temperatures and frequencies and analysed based on the structure available in different parts of bamboo.

\section{Experimental}

\subsection{Materials}

The bamboo (Dendrocalamus strictus) used in this study was collected from Sehore, India. Density of the bamboo was $0.66 \mathrm{~g} / \mathrm{cc}$ at $9 \%$ moisture content. 


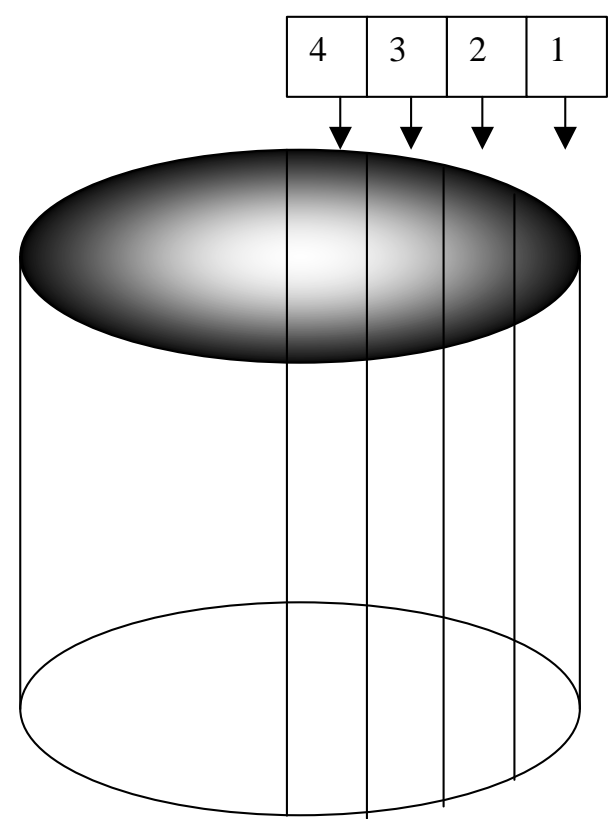

Figure 1. Schematic diagram of bamboo gradient.

\subsection{Sample preparation}

Different strips at fixed distance from the outer periphery to centre were cut from the bamboo stem as shown in figure 1. Test samples were cut from these strips. Uniformity of surfaces was obtained by polishing the sample by using a polishing cloth. Both sides of the sample were coated by using an air drying type graphite conducting paint before dielectric measurements.

\subsection{Testing}

2.3a A.C. conductivity: Effect of temperature and frequency variation on a.c. conductivity was determined by using a 4274 A Multi-Frequencies LCR meter. Electrical measurements were carried out in the temperature range from $24-120^{\circ} \mathrm{C}$ and in the frequency range $4-100 \mathrm{kHz}$.

$\sigma_{\text {a.c. }}$ was calculated using the following relation

$$
\sigma_{\text {a.c. }}=\varepsilon_{0} \omega \varepsilon^{\prime} \tan \delta
$$

where $\varepsilon_{0}$ is the permittivity of the free space $(8.85 \times$ $\left.10^{-12} \mathrm{Fm}^{-1}\right)$, tan $\delta$ the dielectric dissipation factor, $\varepsilon^{\prime}$ the dielectric constant and $\omega$ the angular frequency, which is equal to $2 \pi f$.

2.3b Density: For this test, different strips of bamboo were cut and polished into the circular pellet form of $2 \mathrm{~mm}$ thick and $10 \mathrm{~mm}$ in diameter from the outer periphery to the centre. The density was determined by dividing weight with volume of the bamboo having $9 \mathrm{wt} \%$ moisture at $24^{\circ} \mathrm{C}$.

\section{Results and discussion}

Figure 1 shows the schematic diagram of bamboo section, slicing done is shown from outer most surface to centre core of bamboo, slices are numbered as samples 1, 2, 3, and 4 , respectively.

Figures 2(a)-(d) show the variation of a.c. conductivity with temperature for bamboo samples 1-4 at 4, 10, 20, 40 and $100 \mathrm{kHz}$ frequencies.

Figure 2(a) shows the effect of temperature on a.c. conductivity for bamboo in sample 1 in the temperature range $24-120^{\circ} \mathrm{C}$ at $4,10,20,40$ and $100 \mathrm{kHz}$ frequencies. It was observed that a.c. conductivity initially increases with increasing temperature $\left(24-55^{\circ} \mathrm{C}\right)$ and frequencies and then suddenly decreases with respect to temperature variation (from $40-55^{\circ} \mathrm{C}$ ) at all the frequencies. An interesting observation in a.c. conductivity with temperature behaviour is that at higher temperatures, difference in a.c. conductivity values, between all frequencies decreased, as compared to low temperatures. In figures 2(b)-(d) difference in a.c. conductivity values at low temperature increased. Another unique feature in a.c. conductivity temperature dependence is that phase change temperature shifts towards higher temperature side on increasing the frequency in sample 1 . Phase change occurs at 84, 88, 90, 92 and $94^{\circ} \mathrm{C}$ corresponding to $4,10,20,40$ and $100 \mathrm{kHz}$, respectively.

Figure 3 shows the effect of temperature on a.c. conductivity for bamboo samples 1-4 in the temperature range $24-120^{\circ} \mathrm{C}$ at $4 \mathrm{kHz}$ frequencies. It was observed that a.c. conductivity value increases initially thereafter decreases with respect to temperature variation for all the samples. In this case, sharpness of the peak decreases with the distance from the inner centre to the skin surface. Maximum sharp peak is observed in case of sample 4. Another interesting observation in a.c. conductivity with temperature behaviour is that it increases with distance from outer most surface to the centre of the bamboo. Reversal of a.c. conductivity trend for sample 4 is observed at $52^{\circ} \mathrm{C}$. Maximum a.c. conductivity at room temperature is observed for centre and minimum a.c. conductivity at outer surface of the bamboo. The outer most surface has minimum moisture as compared to centre of the bamboo. At $120^{\circ} \mathrm{C}$ temperature the maximum a.c. conductivity is observed at the outer surface while minimum is observed at centre of the bamboo. Intermediate samples 2 and 3 have intermediate values. At more than $100^{\circ} \mathrm{C}$ temperature all the specimens become dry. There is a transition zone around $90^{\circ} \mathrm{C}$ where sudden change occurs.

Bamboo stem is composed of three parts: bamboo skin, bamboo timber and pith. Bamboo skin is the outermost part of cross-section of stem wall, where no vascular bundles are seen. Pith is the part of stem wall next to bamboo cavity and it does not contain vascular bundles tool. Bamboo timber is the part between skin and pith. Vascular bundles are observed on its cross-section; among vascular 

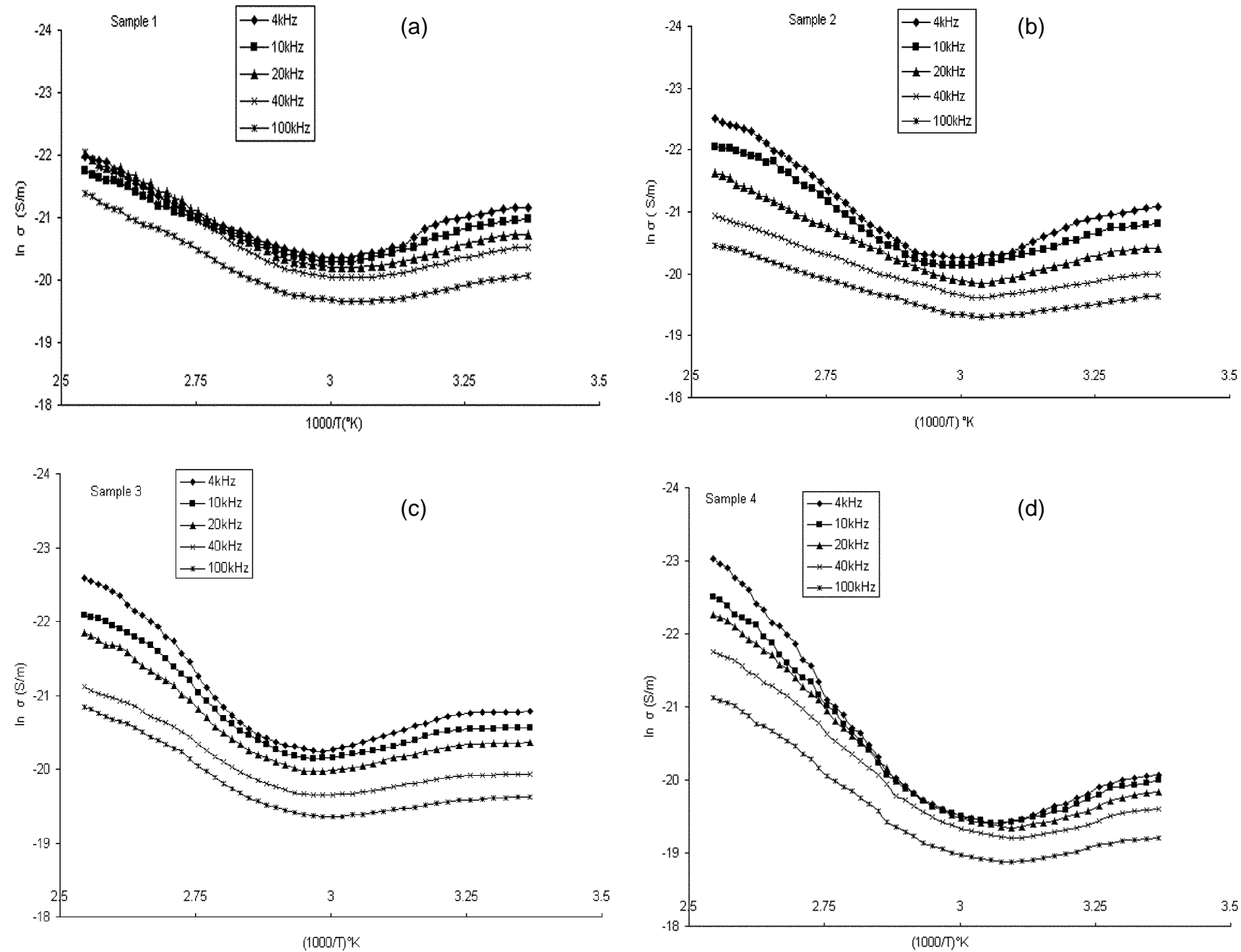

Figure 2. (a) Variation of $\ln \sigma_{\text {a.c. }}$ conductivity vs temperature for bamboo sample 1 at 4, 10, 20, 40 and $100 \mathrm{kHz}$ frequencies, (b) variation of $\ln \sigma_{\text {a.c. }}$ conductivity vs temperature for bamboo sample 2 at $4,10,20,40$ and $100 \mathrm{kHz}$ frequencies, (c) variation of $\ln \sigma_{\text {a.c. }}$ conductivity vs temperature for bamboo sample 3 at 4,10,20, 40 and $100 \mathrm{kHz}$ frequencies and (d) variation of ln $\sigma_{\text {a.c. }}$ conductivity vs temperature for bamboo sample 4 at 4,10,20, 40 and $100 \mathrm{kHz}$ frequencies.

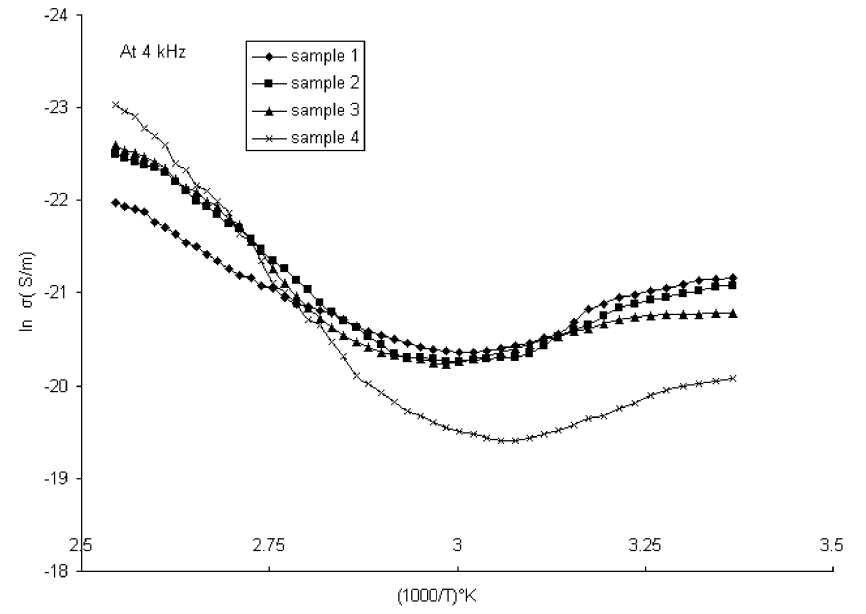

Figure 3. Variation of $\ln \sigma_{\text {a.c. }}$ conductivity vs temperature for bamboo (samples 1,4$)$ at $4 \mathrm{kHz}$ frequencies.

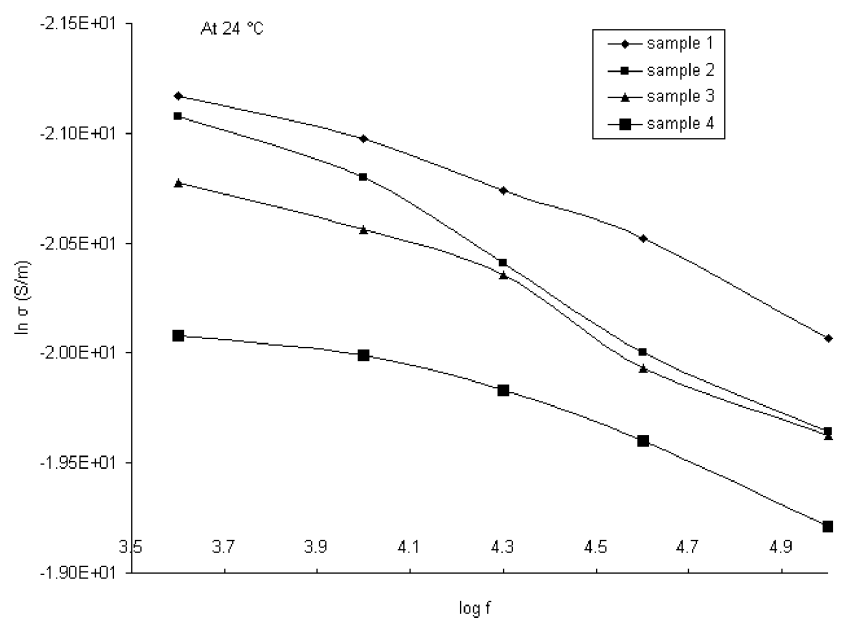

Figure 4. Variation of $\ln \sigma_{\text {a.c. }}$ conductivity vs $\log f$ for bamboo (samples 1,4$)$ at $24^{\circ} \mathrm{C}$. 


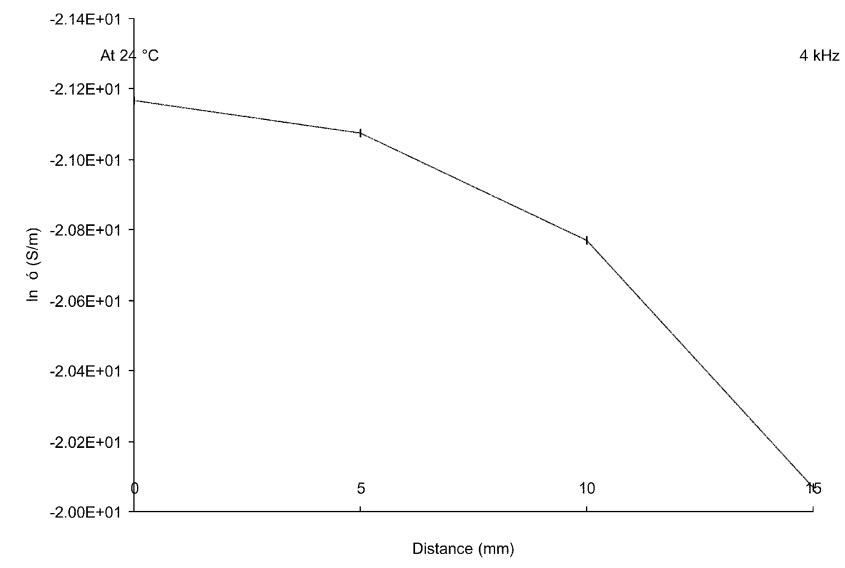

Figure 5. Variation of $\ln \sigma_{\text {a.c. }}$ conductivity vs distance for bamboo gradient at $4 \mathrm{kHz}$ frequencies.

bundles are fundamental tissues. The density of vascular bundles decreases from outer side of stem wall to inner side. The outer part where vascular bundles are dense is called bamboo green, while the inner part where vascular bundles are rare is called bamboo yellow.

Figure 4 shows the effect of frequencies on a.c. conductivity for bamboo samples 1 to 4 . Maximum a.c. conductivity values were observed at the inner (towards pith) most of the bamboo. These maximum a.c. conductivity values decrease from inner most to the outer most surface of the bamboo at all the frequencies at $24^{\circ} \mathrm{C}$, because sample no. 1 has minimum lignin, volatile contents and maximum cellulose content. Sample no. 4 has maximum lignin, volatile contents, and moisture content. In samples 1-4 there exist a gradient increase of lignin and gradient decrease of cellulose and hence there is a gradual increase in a.c. conductivity.

Figure 5 shows the variation of a.c. conductivity with distance for bamboo samples $1-4$ at $4 \mathrm{kHz}$ frequencies. It is observed that the a.c. conductivity increases with distance from outer most surface to the centre of the bamboo.

\section{Conclusions}

(I) Gradient a.c. conductivity characteristics in bamboo is observed.

(II) Two-phase a.c. conductivity behaviour exists in bamboo. (III) A.C. conductivity increased with distance from skin to the inner most centre of the bamboo. At $10 \mathrm{~mm}$ distance sudden increase in a.c. conductivity is found.

(IV) A.C. conductivity increased with increase of temperature and frequencies and gave a peak.

\section{Acknowledgement}

Authors wish to acknowledge that this work is carried out under CTSM, CMM-0022 project.

\section{References}

Chia L H L, Chua P H, Hon Y S and Lee E 1986 Int. J. Radiation Applications and Instrumentation C27 207

Jain S, Jindal U C and Kumar R 2005 J. Mater. Sci. (in press)

Jindal U C 1986 J. Comp. Mater. 2019

Khan M A, Blriss K M and Wang W 1991 Inter. J. Radiation Applications and Instrumentation C Radiation Phys. \& Chem. 38303

Kulkarni A G, Satyanarayana K G and Rohatgi P K $1981 \mathrm{~J}$. Mater. Sci. 161719

Kulkarni A G, Satyanarayana K G and Sukumaran K $1983 \mathrm{~J}$. Mater. Sci. 182390

Roe P S and Ansell M P 1985 J. Mater. Sci. 204015

Shah A N and Lakkad S L 1981 Fibre Sci. Technol. 1541

Shin F G, Xian X, Zheng J W P and Yipp M W 1989 J. Mater. Sci. 243483

White N M and Ansell M P 1983 J. Mater. Sci. 181549 